\title{
MicroRNA-212 inhibits the proliferation and invasion of human renal cell carcinoma by targeting FOXA1
}

\author{
ZHIGANG TONG $^{1}$, XIANFENG MENG ${ }^{1}$, JINSONG WANG $^{1}$ and LIXIN WANG $^{2}$ \\ ${ }^{1}$ Department of Urinary Surgery, The Affiliated Hospital, Changchun University of Chinese Medicine; \\ ${ }^{2}$ Department of Medical Insurance, Jilin Academy of Chinese Medicine Sciences, Changchun, Jilin 130021, P.R. China
}

Received July 7, 2016; Accepted August 31, 2017

DOI: $10.3892 / \mathrm{mmr} .2017 .7956$

\begin{abstract}
MicroRNA-212 (miR-212) has been observed to be significantly deregulated in various types of human cancer. However, the clinical significance of miR-212 and the associated molecular signaling pathways involved in the progression of renal cell carcinoma ( $\mathrm{RCC}$ ) remain unclear. In the present study, miR-212 expression was significantly downregulated in RCC tissues compared with adjacent non-tumor tissues. Clinical association analysis indicated that low expression of miR-212 was prominently associated with large tumor size, advanced tumor, nodes, metastasis stage and lymph node metastasis. In vitro studies revealed that upregulation of miR-212 inhibited cell proliferation, migration and invasion, and induced apoptosis in Caki-1 cells. Forkhead box protein A1 (FOXA1) was identified as a direct target of miR-212 in RCC cells via luciferase reporter assays and western blotting. In addition, FOXA1 expression was upregulated in RCC tissues compared with adjacent noncancerous tissues. An inverse correlation between FOXA1 and miR-212 expression was observed in RCC tissues. Notably, FOXA1 overexpression partially rescued miR-212-mediated inhibition of cell proliferation, migration and invasion in RCC cells. These results suggested that miR-212 suppresses RCC proliferation and invasion by modulating FOXA1, suggesting that miR-212 may have potential as a therapeutic target in RCC.
\end{abstract}

\section{Introduction}

Renal cell carcinoma (RCC) is the most common type of kidney tumor and the most lethal urologic tumor (1). Despite increased early detection of RCC and more frequent surgery, the prognosis remains poor for locally advanced and metastatic cases of RCC $(2,3)$. Therefore, understanding the underlying

Correspondence to: Professor Lixin Wang, Department of Medical Insurance, Jilin Academy of Chinese Medicine Sciences, 1745 Gongnong Road, Chaoyang, Changchun, Jilin 130021, P.R. China

E-mail: wanglixin5421@sina.com

Key words: microRNA, microRNA-212, renal cell carcinoma, forkhead box protein A1, proliferation molecular mechanisms of RCC progression is required, for the development of a novel therapy against advanced RCC.

MicroRNAs (miRNAs/miRs) are a class of small, single stranded, non-coding RNA molecules of 19-25 nucleotides that bind to the 3 ' untranslated regions (UTR) of specific target mRNAs, leading to direct mRNA degradation or translational repression (4). miRNAs may regulate multiple target genes and pathways simultaneously and are involved in regulation of various biological processes, including cell growth, migration, invasion apoptosis, metabolism and cellular differentiation $(5,6)$. Accumulating evidence has suggested that miRNAs may function as tumor suppressors or oncogenes in various types of cancer by directly targeting tumor suppressor genes or oncogenes $(7,8)$. Recent studies demonstrated that a number of miRNAs serve a crucial role in modulating RCC progression (9-11).

miR-212, located at chromosome 17p13.3 (12), has been demonstrated to be deregulated in various types of human cancer, including pancreatic cancer (13), lung cancer (14), prostate cancer (15), cervical cancer (16), glioblastoma (17), ovarian cancer (18) and hepatocellular carcinoma (19). However, the clinical significance of miR-212 and the associated molecular signaling pathways involved in the progression of RCC remain poorly understood. Therefore, the aim of the present study was to investigate the role and underlying molecular mechanism of miR-212 in RCC. Results revealed that miR-212 was downregulated in RCC tissues and cell lines, and that miR-212 functioned as a tumor suppressor in RCC by suppressing cell proliferation, migration and invasion, and inducing cell apoptosis. A direct target, forkhead box protein A1 (FOXA1), was confirmed, which mediated the effects of miR-212. These results may aid the development of an effective therapeutic strategy for RCC.

\section{Materials and methods}

Clinical samples. A total of 48 RCC samples and adjacent non-tumor tissues (ANT; $>3 \mathrm{~cm}$ from the margin of resection) were collected from patients including 20 males and 28 females, who underwent resection of their primary RCC in the Department of General Surgery at The Affiliated Hospital of Changchun University of Chinese Medicine (Changchun, China) between April 2012 and December 2014. Following surgical resection, all samples were immediately stored in 
liquid nitrogen until RNA or protein extraction. The clinicopathological data are listed in Table I. No patients received chemotherapy or radiotherapy prior to surgery. All samples were obtained with informed consent from each patient and the study was approved by the Medicine Ethics Committee of Changchun University of Chinese Medicine.

Cell lines and transfection. A total of three clear cell RCC cell lines (786-O, ACHN and Caki-1) $(20,21)$, a papillary RCC cell line Caki-2 (22) and a human renal proximal tubule epithelial cell line (HK-2) were purchased from the Type Culture Collection of the Chinese Academy of Sciences (Shanghai, China), and were cultured in Dulbecco's modified Eagle medium (DMEM; Gibco; Thermo Fisher Scientific, Inc., Waltham, MA, USA) supplemented with $10 \%$ fetal calf serum (FCS; Invitrogen; Thermo Fisher Scientific, Inc.), $100 \mathrm{IU} / \mathrm{ml}$ penicillin and $100 \mathrm{IU} / \mathrm{ml}$ streptomycin at $37^{\circ} \mathrm{C}$ in an atmosphere containing $5 \% \mathrm{CO}_{2}$.

An miR-212 mimic (5'-CCGGCACUGACCUCUGAC AAU-3') and corresponding negative control (Ctrl; 5'-GUCCTU GCUCGAGCGAGGUGA-3') mimic were purchased from GeneCopoeia, Inc. (Rockville, MD, USA). Plasmids carrying human FOXA1 were purchased from OriGene Technologies, Inc. (Rockville, MD, USA; cat. no. SC108256). Cells were seeded in a six-well plate at a density of $1 \times 10^{3}$ cells/well and transfected with miR-212 (100 nM), miR-Ctrl $(100 \mathrm{nM})$ or FOXA1 overexpression plasmid (100 ng) using Lipofectamine 2000 (Invitrogen; Thermo Fisher Scientific, Inc.) according to the manufacturer's protocol, and transfected cells were cultured for 1-3 days before the cell proliferation, migration, invasion and apoptosis assays.

RNA extraction and reverse transcription-quantitative polymerase chain reaction ( $R T-q P C R)$. Total RNA was isolated from cultured cells and frozen fresh tissues using TRIzol (Invitrogen; Thermo Fisher Scientific, Inc.). To quantify miR-212, cDNA was synthesized using the TaqMan miRNA Reverse Transcription kit (Thermo Fisher Scientific, Inc.), and quantified using the TaqMan Human MicroRNA Assay kit (Thermo Fisher Scientific, Inc.) and the ABI 7900 Sequence Detection system (Life Technologies; Thermo Fisher Scientific, Inc.). Primers for miR-212 and U6 (GeneCopoeia, Inc.) were used as follows: miR-212 forwards, 5'-CGCTAACAGTCTCCAGTC-3' and reverse, 5'-GTGCAG GGTCCGAGGT-3'; U6 forwards, 5'-TGCGGGTGCTCG CTTCGGCAGC-3' and reverse, 5'-CCAGTGCAGGGT CCGAGGT-3'. U6 was used as an internal control. U6 small nuclear RNA was used as an internal control. For detection of FOXA1, total RNA was reverse transcribed to cDNA using the Primer Script RT reagent kit (Takara Biotechnology Co., Ltd., Dalian, China), and quantified on the ABI 7900HT Fast Real-Time PCR system using the SYBR Green PCR Master mix (Takara Biotechnology Co., Ltd.) using primers specific for FOXA1 and GAPDH, as described previously (23). GAPDH was used as an internal control. The following PCR conditions were used: Denaturation at $94^{\circ} \mathrm{C}$ for $3 \mathrm{~min}$, followed by 40 cycles of amplification (denaturation at $94^{\circ} \mathrm{C}$ for $15 \mathrm{sec}$, annealing at $60^{\circ} \mathrm{C}$ for $30 \mathrm{sec}$ and extension at $72^{\circ} \mathrm{C}$ for $45 \mathrm{sec}$ ) The comparative $2^{-\Delta \Delta \mathrm{Cq}}$ method was used for relative quantification (24).
Cell proliferation. Transfected cells were seeded in 24-well plates ( 3,000 cells/well), then cell proliferation was determined at different time points $(24,48$ and $72 \mathrm{~h})$ by incubating cells with $0.5 \mathrm{mg} / \mathrm{ml}$ MTT reagent (Sigma-Aldrich; Merck KGaA, Darmstadt, Germany) for $1 \mathrm{~h}$, and dissolved in dimethyl sulfoxide (Sigma-Aldrich; Merck KGaA) for $10 \mathrm{~min}$ at room temperature. The absorbance of samples was measured using a wavelength of $490 \mathrm{~nm}$ with a microplate reader (Bio-Rad Laboratories, Inc., Hercules, CA, USA).

Cell migration and invasion assay. The migratory and invasive capability of RCC cells was determined using Transwell chambers of diameter $6.5 \mathrm{~mm}$ with an $8-\mu \mathrm{m}$ membrane (Corning Incorporated, Corning, NY, USA). A total of $3 \times 10^{4}$ transfected cells suspended in serum-free medium were added to the upper chamber without Matrigel (for migration) or coated with Matrigel (for invasion), and DMEM containing 10\% FCS was used as an attractant in the lower chamber. After $24 \mathrm{~h}$ (for migration) or $48 \mathrm{~h}$ (for invasion), cells remaining in the upper chamber were removed with a sterile swab, whereas migratory or invasive cells in the bottom chamber were fixed with $70 \%$ ethanol for $30 \mathrm{~min}$ at room temperature $\left(20-25^{\circ} \mathrm{C}\right)$ and stained with $0.2 \%$ crystal violet (Sigma-Aldrich; Merck KGaA) for $10 \mathrm{~min}$ at room temperature $\left(20-25^{\circ} \mathrm{C}\right)$. Cell numbers were counted in five randomly selected microscopic fields under a X71 inverted light microscope (Olympus Corporation, Tokyo, Japan) at x200 magnification.

Cell apoptosis assay. Cell apoptosis was determined using an Annexin-V-FLUOS Staining kit (Roche Applied Science, Penzberg, Germany) under a fluorescence activated cell sorting Calibur instrument (BD Biosciences, Franklin Lakes, NJ, USA), following the manufacturer's protocol. The percentage apoptotic cells was calculated using Cell Quest software (version 3.4; BD Biosciences).

Luciferase reporter assay. Prediction of miR-212 targets was performed using three publicly available algorithms: TargetScan (www.targetscan.org), miRanda (www.microrna.org) and PicTar (www.pictar.org). The 3'-UTR sequence of FOXA1 predicted to bind to miR-212 or a mutated sequence within the predicted target sites was synthesized and inserted into the pGL3 control vector (Promega Corporation, Madison, WI, USA) at the $\mathrm{XbaI}$ and FseI sites, and were referred to as wild-type (Wt) FOXA1-3'UTR or mutant (Mut) FOXA1-3'UTR, respectively. For the reporter assay, Caki-1 cells were seeded into 24 -well plates at density of $2 \times 10^{5}$ for $24 \mathrm{~h}$, and then cotransfected with the Wt/Mut FOXA1 3'UTR and miR-212/miR-Ctrl, and the pRL-TK plasmid (Promega Corporation), which was used for internal normalization, using Lipofectamine 2000 (Invitrogen; Thermo Fisher Scientific, Inc.), according to the manufacturer's protocol. Following $48 \mathrm{~h}$ transfection, the cells were harvested, and a Renilla luciferase activity was determined using the Dual-Luciferase ${ }^{\circledR}$ Reporter assay system (Promega Corporation), following the manufacturer's protocol.

Western blotting. Total protein was extracted from cultured cells using a Total Protein Extraction kit (KeyGen Biotech Co., Ltd., Nanjing, China) and was quantified using a bicinchoninic acid 
Table I. Correlation between clinicopathological features and miR-212 expression in RCC tissues.

miR-212 expression

\begin{tabular}{lccc} 
Variables & No. of cases & Low, $(\%)$ & High, n (\%) \\
\hline Age & 23 & $12(52.2)$ & $11(47.8)$ \\
$\quad<55$ years old & 25 & $14(56.0)$ & $11(44.0)$ \\
$\geq 55$ years old & & & 0.714 \\
Gender & 21 & $11(52.4)$ & $10(47.6)$ \\
Male & 27 & $15(55.6)$ & $12(44.4)$ \\
Female & & & $21(71.8)$ \\
TNM stage & 34 & $13(38.2)$ & $1(7.1)$ \\
T1-T2 & 14 & $13(92.9)$ & $19(61.2)$ \\
T3-T4 & & $12(38.7)$ & $3(17.6)$ \\
Tumor size & 31 & $14(82.4)$ & $<0.01$ \\
$<5$ cm & 17 & $13(37.1)$ & $24(62.9)$ \\
$\geq 5$ cm & & $13(100)$ & $0(0)$
\end{tabular}

miR-212, microRNA-212; RCC, renal cell carcinoma; TNM, tumor nodes metastasis.
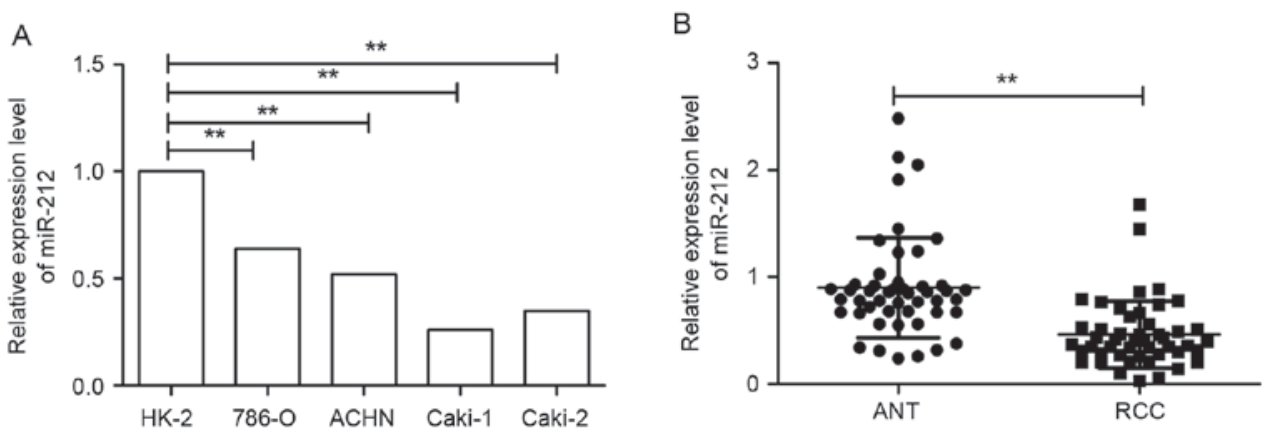

Figure 1. miR-212 expression is downregulated in RCC cell lines and tissues. (A) Relative expression of miR-212 was measured in four RCC cell lines (786-O, ACHN Caki-1 and Caki-2) and the human renal proximal tubule epithelial cell line (HK-2) by RT-qPCR. (B) Relative expression of miR-212 was determined in 48 pairs of RCC tissues sample and ANT using RT-qPCR. ${ }^{* *} \mathrm{P}<0.01$. ANT, adjacent non-tumor tissues; RT-qPCR, reverse transcription-quantitative polymerase chain reaction; miR-212, microRNA-212; RCC, renal cell carcinoma.

protein assay kit (Boster Biological Technology, Pleasanton, CA, USA). A total of $30 \mu \mathrm{g}$ protein/lane was separated using SDS-PAGE on $10 \%$ gels (Bio-Rad Laboratories, Inc.) and then transferred onto nitrocellulose membranes (Bio-Rad Laboratories, Inc.). The membrane was blocked with 5\% non-fat milk (Sigma-Aldrich; Merck $\mathrm{KGaA}$ ) for $2 \mathrm{~h}$ at room temperature, and then incubated with the following antibodies: Mouse monoclonal anti-human FOXA1 (1:1,000; cat no: sc-101058; Santa Cruz Biotechnology, Inc., Dallas, TX, USA) and mouse monoclonal anti-human GAPDH (1:5,000; cat no. sc-365062; Santa Cruz Biotechnology, Inc.) antibodies were incubated overnight at $4^{\circ} \mathrm{C}$. The blots were incubated with polyclonal goat anti-mouse horseradish peroxidase-conjugated immunogloblin G (1:5,000; cat. no. sc-2005; Santa Cruz Biotechnology, Inc.) for $2 \mathrm{~h}$ at room temperature $\left(20-25^{\circ} \mathrm{C}\right)$ and visualized using the enhanced chemiluminescence system (Thermo Fisher Scientific, Inc.). GAPDH was used to normalize the expression levels of the target protein.

Statistical analysis. Results are expressed as the mean \pm standard deviation from $\geq 3$ independent experiments. Statistical analysis was performed using SPSS software (version 17; SPSS, Inc., Chicago, IL, USA) and GraphPad Prism version 5.0 software (GraphPad Software, Inc., La Jolla, CA, USA). An independent t-test was used to compare the differences between two groups. One-way analysis of variance followed by the Bonferroni post-hoc test was performed to compare the differences between three or more groups. Correlations between miR-212 expression and FOXA1 were determined by Spearman's rank correlation coefficient. 

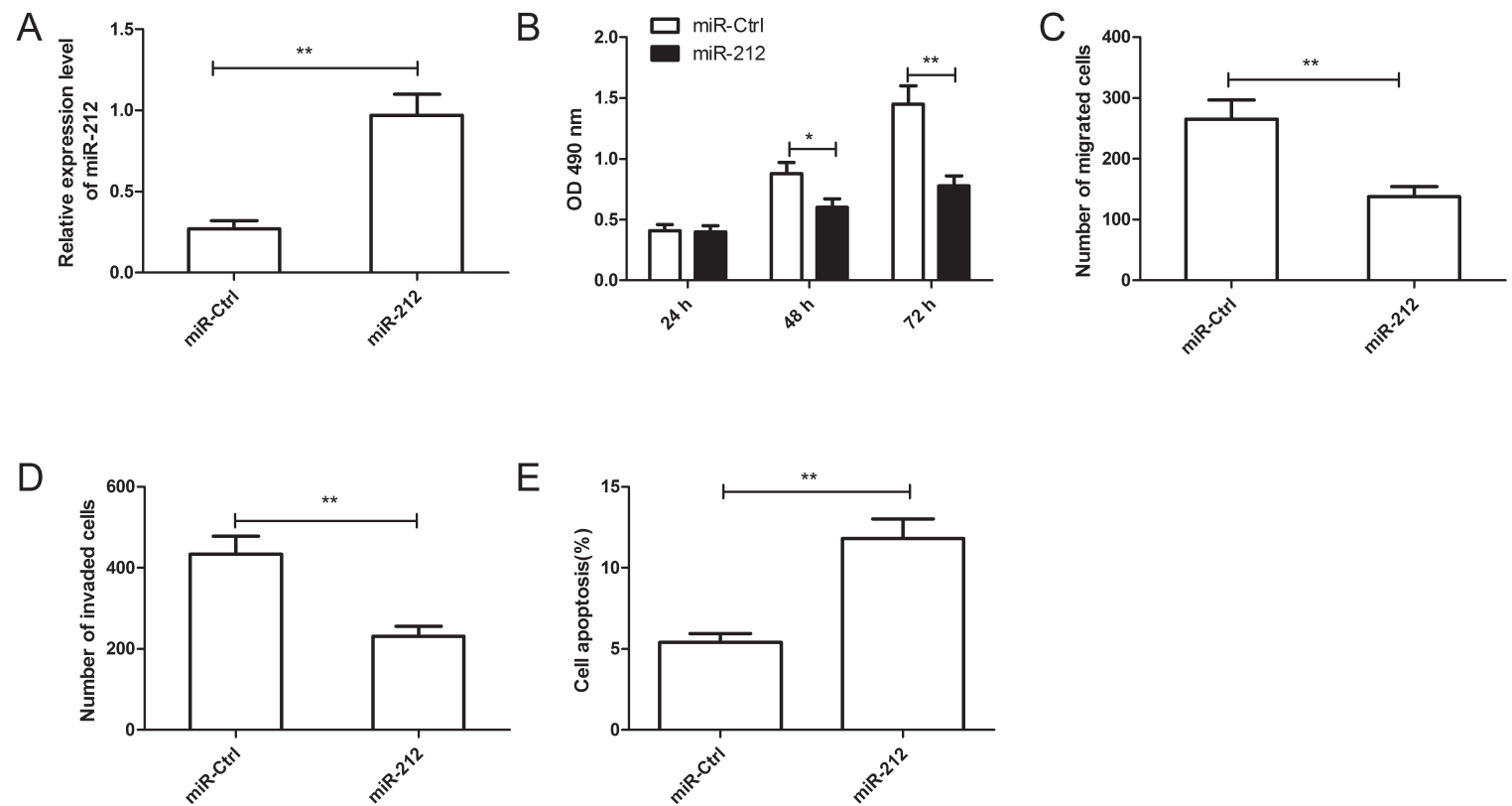

Figure 2. miR-212 inhibits RCC cell proliferation, migration and invasion, and induces apoptosis. (A) Relative expression of miR-212 was measured in Caki-1 cells transfected with miR-212 or miR-Ctrl by reverse transcription-quantitative polymerase chain reaction. (B) Cell proliferation, (C) migration, (D) invasion and (E) apoptosis was determined in Caki-1 cells transfected with miR-212 or miR-Ctrl. ${ }^{*} \mathrm{P}<0.05,{ }^{* *} \mathrm{P}<0.01$. miR-212, microRNA-212; RCC, renal cell carcinoma; miR-Ctrl, microRNA-control.

A

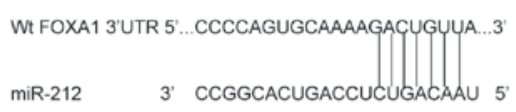

MUt FOXA1 3'UTR 5'...CCCCAGUGCAAAAGUGUCUAA ...3'

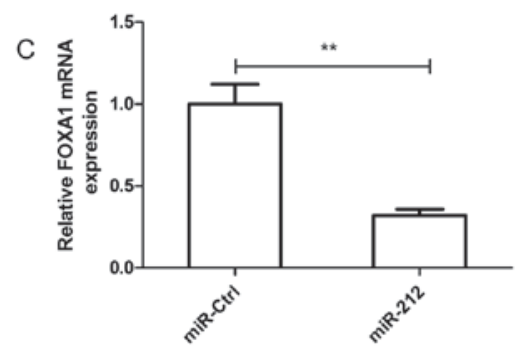

$\mathrm{B}$
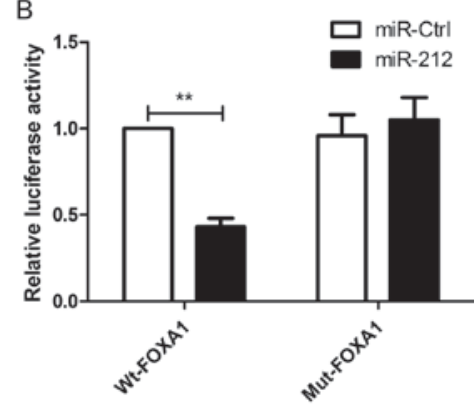

$\mathrm{D}$

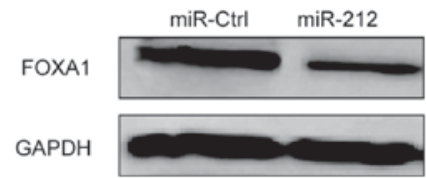

Figure 3. FOXA1 is a direct target of miR-212 in RCC. (A) The target prediction software (TargetScan) indicated that miR-212 bound to the sequences in the 3'UTR of FOXA1. The mutant miR-212 binding site was generated in the complementary site for the seed region of miR-212. (B) Luciferase reporter assays in Caki-1 cells following cotransfection with wild-type or mutant 3'UTR of FOXA1 and miR-212 mimic or miR-Ctrl. (C) Reverse transcription-quantitative polymerase chain reaction and (D) western blot analysis of FOXA1 expression in Caki-1 cells transfected with miR-212 mimic or miR-Ctrl. GAPDH was used as an internal control. ${ }^{* *} \mathrm{P}<0.01$. Wt, wild type; Mut, mutant type; FOXA1, forkhead box protein A1; miR-212, microRNA-212; 3'UTR, 3'untranslated region; miR-Ctrl, microRNA-control; RCC, renal cell carcinoma.

$\mathrm{P}<0.05$ was considered to indicate a statistically significant difference.

\section{Results}

miR-212 expression is downregulated in RCC cell lines and tissues. To determine the expression status of miR-212 in RCC, the expression levels of miR-212 in four RCC cell lines was analyzed by RT-qPCR. Reduced expression levels of miR-212 were observed in all four RCC cell lines compared with the human renal proximal tubule epithelial cell line
$(\mathrm{P}<0.01$; Fig. 1A). The expression of miR-212 in 48 pairs of HCC tissues and ANT was then measured. The expression of miR-212 in RCC tissues was significantly decreased compared with that in matched ANT ( $\mathrm{P}<0.01 ;$ Fig. 1B). All RCC samples were divided into miR-212 low-expression group $(n=26)$ and high-expression group $(\mathrm{n}=22)$ according to the cut-off value, which was defined as the mean value $(0.446)$ in all RCC samples. miR-212 expression was significantly associated with tumor size, tumor, nodes, metastasis (TNM) stage and lymph node metastasis (all $\mathrm{P}<0.01$ ); however, miR-212 expression was not significantly associated with gender and 
A

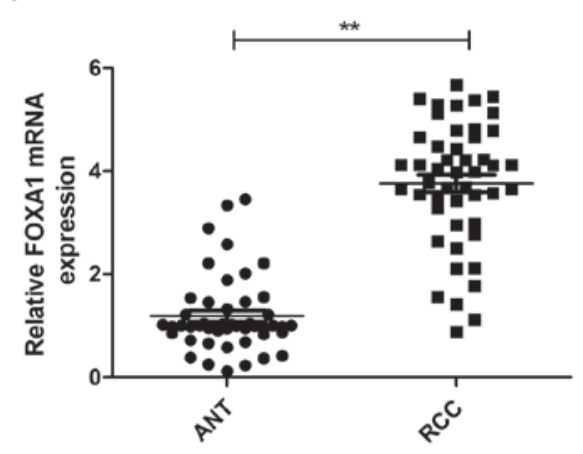

B

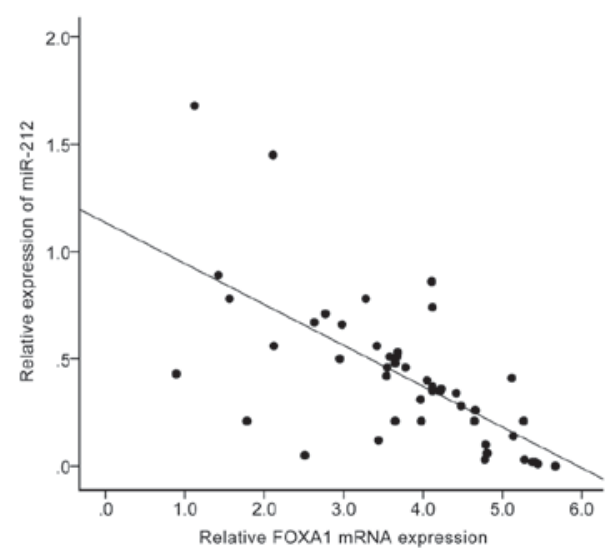

Figure 4. FOXA1 is upregulated, and is inversely correlated with miR-212 in RCC tissues. (A) FOXA1 mRNA expression levels were measured in 48 pairs of RCC tissue samples and ANT using reverse transcription-quantitative polymerase chain reaction. (B) Correlation of miR-212 and FOXA1 expression was determined in human RCC tissues by Spearman's rank correlation coefficient analysis $(\mathrm{n}=48) .{ }^{* *} \mathrm{P}<0.01$. ANT, adjacent non-tumor tissues; FOXA1, forkhead box protein A1; miR-212, microRNA-212; RCC, renal cell carcinoma.
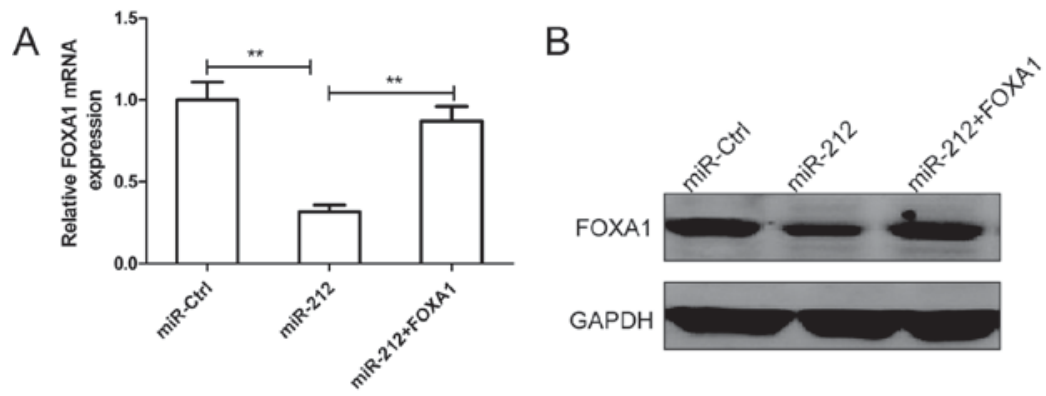

$\mathrm{D}$

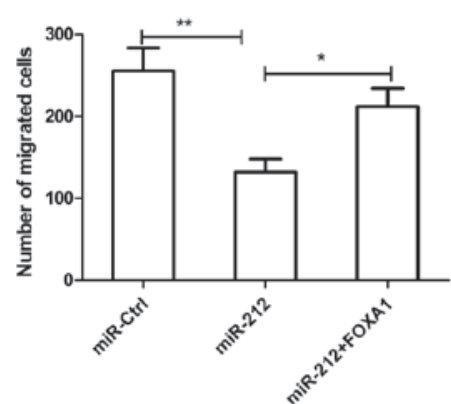

$\mathrm{E}$

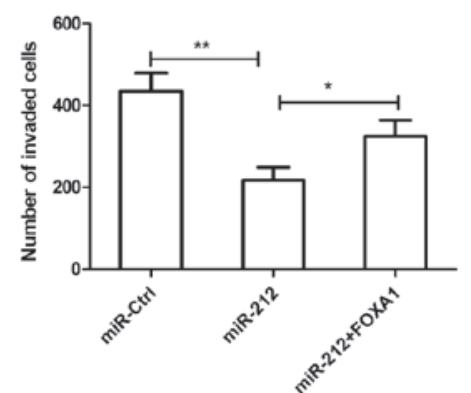

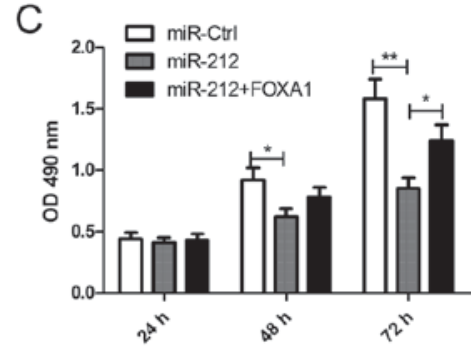

$\mathrm{F}$

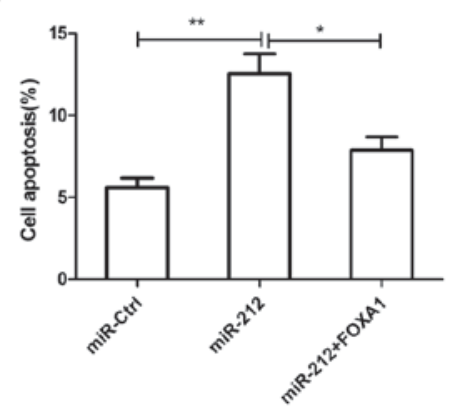

Figure 5. FOXA1 overexpression reverses the inhibitory effects of miR-212 in RCC cells. Caki-1 cells were cotransfected with miR-212 mimic and FOXA1 overexpression plasmid, together with miR-Ctrl, and then subjected to the following examination. (A) reverse transcription-quantitative polymerase chain reaction and (B) western blotting were performed to analyze the FOXA1 mRNA and protein expression levels in Caki-1 cells. GAPDH was used for internal control. (C) Cell proliferation, (D) migration, (E) invasion and (F) apoptosis were determined in Caki-1 cells. "P<0.05, ${ }^{\text {*** }} \mathrm{P}<0.01$. FOXA1, forkhead box protein A1; miR-212, microRNA-212; RCC, renal cell carcinoma; miR-Ctrl, microRNA-control.

age (Table I). These results suggested that miR-212 may serve a key role in the development and progression of RCC.

miR-212 inhibits RCC cell proliferation, migration and invasion, and induces apoptosis. To further investigate the biological function of miR-212 in RCC, miR-212 mimic or miR-Ctrl was transfected into Caki-1 cells (Fig. 2A). As measured by RT-qPCR, the miR-212 mimic significantly increased the level of miR-212 in Caki-1 cells compared with the miR-Ctrl ( $\mathrm{P}<0.01$; Fig. 2A). The MTT assay demonstrated that overexpression of miR-212 significantly inhibited cell proliferation of Caki-1 cells after 48 and $72 \mathrm{~h}$ compared with the miR-Ctrl (Fig. 2B). A Transwell chamber was then used to investigate the roles of miR-212 in cell migration and invasion in Caki-1 cells. Results revealed that overexpression of miR-212 significantly inhibited the migratory and invasive capabilities in Caki-1 cells compared with the miR-Ctrl (Fig. 2C and D). In addition, compared with the miR-Ctrl, overexpression of miR-212 significantly induced apoptosis in Caki-1 cells (Fig. 2E). These results suggested that miR-212 may serve a suppressive role in RCC.

FOXAl is a functional target of miR-212 in RCC. To investigate the underlying molecular mechanism by which 
miR-212 inhibits RCC tumor growth, analysis of bioinformatic databases (TargetScan, PicTar and miRanda) were used to predict putative miR-212 targets. Target prediction analysis revealed that the 3'-UTR of FOXA1 mRNA contained the complementary sequence of miR-212 at position 164-171 (Fig. 3A). To further assess whether FOXA1 is a direct target of miR-212, a luciferase reporter assay was performed. The results demonstrated that miR-212 significantly inhibited the luciferase activity of FOXA1 containing the Wt 3'-UTR, but no alteration in FOXA1 activity was observed with the Mut 3'-UTR, compared with Caki-1 cells transfected with the miR-Ctrl (Fig. 3B). In addition, RT-qPCR and western blotting results confirmed that overexpression of miR-212 resulted in downregulation of FOXA1 expression at the mRNA (Fig. 3C) and protein (Fig. 3D) level in Caki-1 cells.

FOXA1 is inversely correlated with miR-212 in RCC tissues. FOXA1 mRNA expression in 48 pairs of RCC tissue samples and ANT was investigated by RT-qPCR. FOXA1 mRNA expression was increased in RCC specimens compared with ANT (Fig. 4A). Using Spearman's rank correlation coefficient analysis, an inverse correlation between miR-212 and FOXA1 mRNA expression levels was confirmed in $48 \mathrm{RCC}$ tissues $(r=-0.667 ; \mathrm{P}<0.0001 ;$ Fig. 4B).

FOXA1 overexpression reverses the inhibitory effects of miR-212 in RCC cells. To determine whether FOXA1 mediates miR-212 in cell proliferation, migration and invasion, a rescue experiment was performed. Caki-1 cells were cotransfected with miR-212 mimic and FOXA1 overexpression plasmid, in parallel with controls (miR-Ctrl). The expression of FOXA1 was confirmed by RT-qPCR (Fig. 5A) and western blotting (Fig. 5B). In addition, it was revealed that overexpression of FOXA1 restored cell proliferation (Fig. 5C), migration (Fig. 5D) and invasion (Fig. 5E) in Caki-1 cells that were inhibited by miR-212. The increased apoptotic rate induced by miR-212 in Caki-1 cells was also reversed by FOXA1 overexpression (Fig. 5F).

\section{Discussion}

Accumulating evidence has identified a number of miRNAs with aberrant expression in RCC tissues or cell lines, which are involved in the initiation and progression of RCC as a tumor suppressor or oncogene (9-11). Therefore, investigating the function of miRNAs specifically involved in RCC development and progression may contribute to the understanding RCC carcinogenesis, and provide novel diagnostics and therapeutic targets for this disease. In the present study, the results revealed that miR-212 expression was downregulated in RCC cells lines and tissues. Furthermore, reduced expression of miR-212 was associated with poor prognostic features of RCC. Overexpression of miR-212 suppressed the cell proliferation, migration and invasion, and induced cell apoptosis. These results suggested that miR-212 may be a novel potential therapeutic strategy for RCC.

miR-212 has been reported to be downregulated and act as a tumor suppressor in several types of cancers, including lung cancer (14), cervical cancer (16), glioblastoma (17), ovarian cancer (18), hepatocellular carcinoma (19) and gastric carcinoma (25). However, several other studies have suggested that miR-212 exhibits oncogenic roles in pancreatic cancer (13), prostate cancer (15) and colorectal cancer (26). This suggests that the biological functions of miR-212 are cancer type specific, partly resulting from the different cellular contexts of various types of cancer. In the present study, the expression and biological function of miR-212 in RCC was investigated. miR-212 expression in RCC tissues was significantly downregulated as compared with that in adjacent non-tumor tissues. In addition, results demonstrated that reduced expression of miR-212 was associated with TNM stage and lymph node metastasis. miR-212 significantly suppressed RCC proliferation, migration and invasion, and induced cell apoptosis. These results suggested that miR-212 has a possible tumor suppressor role in RCC.

To further investigate the underlying molecular mechanisms by which miR-212 exerts its anti-tumor effect on RCC cells, identification of its downstream functional targets is necessary. Using three public databases (TargetScan,PicTar, and miRanda), it was predicted that FOXA1 was a direct target of miR-212. FOXA1, a member of the FOXA gene family, has been reported to serve an important regulatory role in proliferation, apoptosis and the cell cycle (27). FOXA1 has been demonstrated to be upregulated in various types of cancer, including RCC (28), breast cancer $(29)$, hepatocellular carcinoma $(18,19)$, prostate cancer (30) and gastric cancer (31). In addition, FOXA1 may promote cancer cell proliferation and inhibit apoptosis partly by upregulating Yes-associated protein expression, suggesting its oncogenic role in various types of cancer $(27,31,32)$. FOXA1 has been reported to be a target of miR-212 in hepatocellular carcinoma (19,23); however, the interaction between miR-212 and FOXA1 has not been experimentally validated in RCC. In the present study, it was confirmed that FOXA1 was a direct downstream target of miR-212 as evidenced by the observation that ectopic expression of miR-212 reduced luciferase activity of the FOXA1 promoter and miR-212 overexpression downregulated FOXA1 expression. An inverse correlation between the expression of miR-212 and FOXA1 mRNA expression was observed in RCC tissues. Overexpression of FOXA1 expression partly abrogated the functional effect of miR-212 on RCC cell proliferation, migration, invasion and apoptosis. These data may suggest that miR-212 partly exerts it antitumor role in RCC by targeting FOXA1.

In conclusion, the present study revealed that miR-212 is downregulated in RCC cell lines and tissues. Low expression of miR-212 was prominently associated with large tumor size, advanced TNM stage and lymph node metastasis. It was also demonstrated that miR-212 overexpression significantly inhibited cell proliferation, migration and invasion, and induced cell apoptosis in RCC cells by suppressing FOXA1 expression. These results suggested that miR-212 may potentially act as a clinical biomarker and a therapeutic target for RCC.

\section{References}

1. Siegel R, Ma J, Zou Z and Jemal A: Cancer statistics, 2014. CA Cancer J Clin 64: 9-29, 2014.

2. Chow WH, Dong LM and Devesa SS: Epidemiology and risk factors for kidney cancer. Nat Rev Urol 7: 245-257, 2010.

3. Pantuck AJ, Zisman A and Belldegrun AS: The changing natural history of renal cell carcinoma. J Urol 166: 1611-1623, 2001. 
4. Guo H, Ingolia NT, Weissman JS and Bartel DP: Mammalian microRNAs predominantly act to decrease target mRNA levels. Nature 466: 835-840, 2010

5. Fabian MR, Sonenberg N and Filipowicz W: Regulation of mRNA translation and stability by microRNAs. Ann Rev Biochem 79: 351-379, 2010.

6. Bartel DP: MicroRNAs: Genomics, biogenesis, mechanism, and function. Cell 116: 281-297, 2004.

7. Farazi TA, Spitzer JI, Morozov P and Tuschl T: miRNAs in human cancer. J Pathol 223: 102-115, 2011.

8. Garzon R and Marcucci G: Potential of microRNAs for cancer diagnostics, prognostication and therapy. Curr Opin Oncol 24: 655-659, 2012

9. Gu L, Li H, Chen L, Ma X, Gao Y, Li X, Zhang Y, Fan Y and Zhang X: MicroRNAs as prognostic molecular signatures in renal cell carcinoma: A systematic review and meta-analysis. Oncotarget 6: 32545-32560, 2015.

10. Zhang S, Zhang D, Yi C, Wang Y, Wang H and Wang J: MicroRNA-22 functions as a tumor suppressor by targeting SIRT1 in renal cell carcinoma. Oncol Rep 35: 559-567, 2016.

11. Jingushi K, Ueda Y, Kitae K, Hase H, Egawa H, Ohshio I, Kawakami R, Kashiwagi Y, Tsukada Y, Kobayashi T, et al: miR-629 Targets TRIM33 to Promote TGF $/$ Smad Signaling and Metastatic Phenotypes in ccRCC. Mol Cancer Res 13: $565-574,2015$

12. Ucar A, Vafaizadeh V, Jarry H, Fiedler J, Klemmt PA, Thum T, Groner B and Chowdhury K: miR-212 and miR-132 are required for epithelial stromal interactions necessary for mouse mammary gland development. Nat Genet 42: 1101-1108, 2010.

13. Ma C, Nong K, Wu B, Dong B, Bai Y, Zhu H, Wang W, Huang X Yuan Z and Ai K: miR-212 promotes pancreatic cancer cell growth and invasion by targeting the hedgehog signaling pathway receptor patched-1. J Exp Clin Cancer Res 33: 54, 2014.

14. Incoronato M, Urso L, Portela A, Laukkanen MO, Soini Y, Quintavalle C, Keller S, Esteller M and Condorelli G: Epigenetic regulation of miR-212 expression in lung cancer. PLoS One 6: e27722, 2011

15. Yang Y, Jia D, Kim H, Abd Elmageed ZY, Datta A, Davis R, Srivastav S, Moroz K, Crawford BE, Moparty K, et al: Dysregulation of miR-212 promotes castration resistance through hnRNPH1-Mediated regulation of AR and AR-V7: Implications for racial disparity of prostate cancer. Clin Cancer Res 22: 1744-1756, 2016.

16. Zhao JL, Zhang L, Guo X, Wang JH, Zhou W, Liu M, Li X and Tang H: miR-212/132 downregulates SMAD2 expression to suppress the G1/S phase transition of the cell cycle and the epithelial to mesenchymal transition in cervical cancer cells IUBMB Life 67: 380-394, 2015.

17. Liu H, Li C, Shen C, Yin F, Wang K, Liu Y, Zheng B, Zhang W, Hou X, Chen X, et al: MiR-212-3p inhibits glioblastoma cell proliferation by targeting SGK3. J Neurooncol 122: 431-439, 2015.

18. Wei LQ, Liang HT, Qin DC, Jin HF, Zhao Y and She MC: MiR-212 exerts suppressive effect on SKOV3 ovarian cancer cells through targeting HBEGF. Tumour Biol 35: 12427-12434, 2014.
19. Dou C, Wang Y, Li C, Liu Z, Jia Y, Li Q, Yang W, Yao Y, Liu Q and Tu K: MicroRNA-212 suppresses tumor growth of human hepatocellular carcinoma by targeting FOXA1. Oncotarget 6: 13216-13228, 2015

20. Huang JL, Liao Y, Qiu MX, Li J and An Y: Long non-coding RNA CCAT2 promotes cell proliferation and invasion through regulating $\mathrm{Wnt} / \beta$-catenin signaling pathway in clear cell renal cell carcinoma. Tumour Biol 39: 1010428317711314, 2017.

21. Zhu J, Cui L, Xu A, Yin X, Li F and Gao J: MEIS1 inhibits clear cell renal cell carcinoma cells proliferation and in vitro invasion or migration. BMC Cancer 17: 176, 2017.

22. Furge KA, Chen J, Koeman J, Swiatek P, Dykema K Lucin K, Kahnoski R, Yang XJ and Teh BT: Detection of DNA copy number changes and oncogenic signaling abnormalities from gene expression data reveals MYC activation in high-grade papillary renal cell carcinoma. Cancer Res 67: 3171-3176, 2007

23. Tu H, Wei G, Cai Q, Chen X, Sun Z, Cheng C, Zhang L, Feng Y, Zhou H, Zhou B and Zeng T: MicroRNA-212 inhibits hepatocellular carcinoma cell proliferation and induces apoptosis by targeting FOXA1. OncoTargets Ther 8: 2227-2235, 2015.

24. Livak KJ and Schmittgen TD: Analysis of relative gene expression data using real-time quantitative PCR and the 2(-Delta Delta C(T)) method. Methods 25: 402-408, 2001.

25. Jiping Z, Ming F, Lixiang W, Xiuming L, Yuqun S, Han Y, Zhifang L, Yundong S, Shili L, Chunyan C and Jihui J: MicroRNA-212 inhibits proliferation of gastric cancer by directly repressing retinoblastoma binding protein 2. J Cell Biochem 114: 2666-2672, 2013.

26. Meng X, Wu J, Pan C, Wang H, Ying X, Zhou Y, Yu H, Zuo Y, Pan Z, Liu RY and Huang W: Genetic and epigenetic down-regulation of microRNA-212 promotes colorectal tumor metastasis via dysregulation of MnSOD. Gastroenterology 145: 426-436 e1-e6, 2013

27. Bernardo GM and Keri RA: FOXA1: A transcription factor with parallel functions in development and cancer. Biosci Rep 32: 113-130, 2012.

28. Neely BA, Wilkins CE, Marlow LA, Malyarenko D, Kim Y, Ignatchenko A, Sasinowska H, Sasinowski M, Nyalwidhe JO, Kislinger T, et al: Proteotranscriptomic analysis reveals stage specific changes in the molecular landscape of clear-cell renal cell carcinoma. PLoS One 11: e0154074, 2016

29. Shou J, Lai Y, Xu J and Huang J: Prognostic value of FOXA1 in breast cancer: A systematic review and meta-analysis. Breast 27 35-43, 2016.

30. Yang YA and Yu J: Current perspectives on FOXA1 regulation of androgen receptor signaling and prostate cancer. Genes Dis 2: 144-151, 2015.

31. Ren H, Zhang P, Tang Y, Wu M and Zhang W: Forkhead box protein A1 is a prognostic predictor and promotes tumor growth of gastric cancer. OncoTargets Ther 8: 3029-3039, 2015.

32. Augello MA, Hickey TE and Knudsen KE: FOXA1: Master of steroid receptor function in cancer. EMBO J 30: 3885-3894, 2011. 